\title{
O impacto do boca-a-boca online: um estudo sobre a adoção de opiniões em comunidades de consumidores online
}

The impact of eWOM: a study on the adoption of opinions on consumer online communities

\section{Jorge Brantes Ferreira}

PUC-Rio - Pontifícia Universidade Católica do Rio de Janeiro, Rua Marquês de São Vicente, 225, Gávea, Rio de Janeiro, RJ, 22451-900, Brasil, jorgebf@gmail.com

\section{Angilberto Sabino de Freitas}

Unigranrio - Universidade Grande Rio, Rio de Janeiro, RJ, 22775-002, Brasil, angilberto.freitas@gmail.com

\section{Eduardo Rocha Guaraná}

PUC-Rio - Pontifícia Universidade Católica do Rio de Janeiro, Rio de Janeiro, 22451-900, Brasil, eduardo@guarana.name

\section{Thais Vieira de Lima}

Unigranrio - Universidade Grande, Rio de Janeiro, RJ, 22775-002, Brasil, thaisvieiradelima@gmail.com

\section{Juliana Werneck Rodrigues}

PUC-Rio - Pontifícia Universidade Católica do Rio de Janeiro, Rio de Janeiro, 22451-900, Brasil, juwrnck@gmail.com

\section{Cristiane Junqueira Giovannini}

PUC-Rio - Pontifícia Universidade Católica do Rio de Janeiro, Rio de Janeiro, 22451-900, Brasil, mestrekis@gmail.com

\section{Resumo}

A internet causou uma verdadeira revolução no marketing boca-a-boca. Essa comunicação, que antes demandava o contato pessoal, agora é compartilhada globalmente por meio de plataformas virtuais entre indivíduos desconhecidos e geograficamente dispersos. O objetivo deste estudo é avaliar os fatores que afetam a adoção de informações em comunidades de consumidores online. Com uma amostra de 288 respondentes, os dados obtidos foram analisados por meio da modelagem de equações estruturais. Os resultados apontam que a percepção de utilidade das informações se mostrou importante para o processo de adoção das opiniões, sendo a relevância e a confiabilidade da fonte os fatores que mais influenciaram a utilidade percebida.

Palavras-chave: eCommerce, boca-a-boca online, comportamento do consumidor.

\section{Abstract}

Internet has revolutionized word-of-mouth marketing. Once face-to-face, word-of-mouth communications can now be exchanged globally via a multitude of platforms, allowing virtual strangers to share information regardless of geographical boundaries. This study aims to evaluate factors affecting the adoption of information among members of online consumer communities. Structural Equation Modelling (SEM) was used to analyze data from a 288 respondent sample acquired via survey and results indicate that information utility, majorly driven by its relevance and reliability, is crucial for information adoption processes.

Keywords: e-commerce, eWOM, consumer behavior.

\section{Introdução}

A comunicação boca-a-boca é conhecida como uma eficiente ferramenta de marketing, dada a influência que exerce sobre o comportamento do consumidor. Pesquisas apontam que a influência pessoal tem maior impacto sobre as decisões de compra do que mensagens publicitárias (Katz \& Lazarsfeld, 1955). A comunicação boca-a-boca é um poderoso meio de disseminar informações de produtos por estar desprovida de incentivos financeiros. Aspectos como a atratividade, competência e confiabilidade da fonte de informações são igualmente importantes no processo de adoção de uma mensagem. Além disso, contatos pessoais costumam ser percebidos como superiores a mensagens de marketing impessoais (Goldsmith, 2006).

Com o advento da internet, crescem as possibilidades de acesso à informação e à interação entre consumidores e desses com as empresas (Goldsmith, 2006). Essa comunicação ocorre por meio de plataformas online, como blogs, sites de comunidades de consumo, fóruns de discussão, redes sociais e salas de bate-papo
(Goldsmith, 2006; Kozinetz, Valck, Wojnicki \& Wilner, 2010). Nesse contexto, a influência da comunicação interpessoal gera o chamado electronic word-of-mouth (eWOM) ou boca-a-boca online (Goldsmith; Horowitz, 2006; Ya, Vadakkepatt \& Joshi, 2015). Diferente do boca-a-boca tradicional, em que as mensagens são transmitidas por meio do contato pessoal, o eWOM ocorre de forma virtual e no formato textual, geralmente entre indivíduos desconhecidos e geograficamente dispersos (Park \& Lee, 2009).

No contexto do turismo, o eWOM se torna cada vez mais importante, dado o aspecto intangível de uma viagem turística a as expectativas criadas pelos consumidores em relação a esse tipo de serviço (Luo \& Zhong, 2015). E com o crescimento das redes sociais, cada vez mais o conteúdo gerado pelos integrantes das redes se torna uma importante fonte de informações que pode influenciar o processo decisório do indivíduo (Ye, Law, G, \& Chen, (2011). Nesse sentido, principalmente com o crescimento do uso dos smartphones e tablets, informações turísticas nas mídias sociais estão sendo cada vez mais compartilhadas e utilizadas pelo consumidor (Munar \& Jacobsen, 2014). 
Entre as formas mais utilizadas para o eWOM estão as comunidades de consumidores online. Seu uso é simples e não exige conhecimentos específicos, permitindo que qualquer pessoa com acesso à Internet possa publicar ou buscar informações. Além disso, é possível encontrar comunidades de consumidores online relacionadas a praticamente todas as áreas de consumo (HennigThurau, Gwinner, Walsh \& Gremler, 2004). Entre as inúmeras comunidades desse tipo presentes no Brasil, pode-se citar o TripAdvisor, Yelp, Kekanto e Foursquare.

Com o crescimento da importância dessas comunidades virtuais, diversos estudos tem investigado os motivos por trás do engajamento com o eWOM tanto no que diz respeito à produção de informação (Hennig-Thurau et al., 2004; Lee, Cheung, Lim \& Sia, 2006) quanto na busca de informações (Goldsmith \& Horowitz, 2006; Goldsmith, 2006). Aliado a isso, devido às características do setor de turismo, em que as expectativas formadas pelos consumidores envolvem emoções (Luo \& Zhong, 2015), a disseminação, compartilhamento e o uso da informação passa a ter papel crucial no processo de decisão do consumidor. Argumenta-se que entender de que forma a mesma se dissemina e é apropriada pelo consumidor se torna relevante para definir estratégias no nível gerencial, principalmente devido ao impacto e influência que as comunidades virtuais possuem o consumidor de produtos turísticos. Entretanto, no contexto brasileiro, uma busca no portal Spell apontou apenas um trabalho investigando o tema eWOM de forma geral (Vieira, Matos \& Slongo, 2009) e, em particular, nenhum trabalho que investiga o impacto das mídias sociais na disseminação e influência da informação no consumidor de turismo, sugerindo haver uma lacuna de estudos sobre o tema no Brasil. Assim, considerando a importância do impacto das comunidades e mídias sociais no setor de turismo, e que as pesquisas sobre o tema ainda estão em seu estágio embrionário (Michaelidou, Siamagka, \& Christodoulides, 2011), o objetivo deste trabalho é analisar os fatores que afetam a adoção de informações em comunidades online de consumo por usuários de turismo e seu impacto na decisão de compra, para, dessa forma, avaliar como aspectos relacionados às mensagens influenciam o comportamento do indivíduo em adotar a informação.

\section{Revisão da literatura}

\subsection{Comunidade virtuais}

Uma comunidade virtual é qualquer grupo de pessoas que, por meio da internet, compartilha laços em comum sem que haja uma dependência de interação física ou de uma mesma localização geográfica (Mcdonough, 1997), como, por exemplo, uma comunidade de consumidores online.

Hennig-Thurau et al. (2004) argumentam que as comunidades de consumidores online constituem a plataforma mais comum para o uso do eWOM, fornecendo aos consumidores a oportunidade de lerem opiniões de consumo e experiências de outros consumidores, além de permitir que possam divulgar seus próprios relatos. Para justificar o sucesso dessas comunidades, os autores destacam a simplicidade de sua utilização, não exigindo um conhecimento avançado sobre o uso da internet. Além do mais, pode-se encontrar comunidades sobre praticamente qualquer área de consumo.

\subsection{Boca-a-boca online}

O boca-a-boca - electronic word-of-mouth (eWOM) - pode ser definido como sendo qualquer declaração positiva ou negativa feita por potenciais, atuais ou ex-clientes sobre um produto ou empresa, que é disponibilizada para um grande número de pessoas e instituições por meio da Internet (Hennig-Thurau et al., 2004). Cheung, Lee e Rabjohn (2008) descrevem o eWOM como uma extensão da comunicação interpessoal tradicional por meio do uso da Internet.

O eWOM não ocorre apenas quando há contato pessoal (Gelb \& Sundaram, 2002), mas com informações podendo ser transmitidas e recebidas por qualquer pessoa em qualquer parte do mundo e a qualquer tempo (Chan \& Ngai, 2011). Dentre as plataformas onde o eWOM pode ocorrer, estão os sites de avaliação e recomendação, blogs, comunidades de consumo online, fóruns de discussão, redes sociais, bem como salas de bate-papo (Goldsmith, 2006).

Dado o exponencial crescimento das comunidades e redes sociais online e o avanço do e-commerce, o eWOM tem atraído grande atenção nos últimos anos (Ya et al., 2015). Vários estudos tem buscado compreender como o eWOM afeta o comportamento do consumidor. Hennig-Thurau et al. (2004) e Lee et al. (2006) investigaram os motivos pelos quais as pessoas se engajam com o eWOM para publicar informações acerca de produtos e serviços, enquanto Goldsmith e Horowitz (2006), Goldsmith (2006) e Khammash e Griffiths (2011) investigaram os motivos por trás da busca pela informação.

Lou \& Zhong (2015) mostram que a comunicação eWOM via mídias sociais relacionada com viagens de turismo podem ter laços classificados como forte, de força mediana, ou fraco, destacando a importância das relações sociais e redes sociais sobre a eWOM. Argumentam ainda que a eWOM pode ter influência decisiva no processo de decisão do consumidor ao escolher um destino turístico. Já Hudson, Roth, Madden e Hudson (2015) argumentam que relações baseadas em mídia social online levam a resultados desejados, tais como um boca a boca positivo.

Kozinetz et al. (2010) apontam que mensagens e significados não fluem unidirecionalmente, mas são trocados entre os membros pertencentes a mesma rede. Além do mais, pistas de proximidade temporal ampliam o valor de um comentário positivo, aumentando a probabilidade de escolha de um produto avaliado positivamente (Chen \& Lurie, 2013). Já Liang, Ekincia, Occhiocupoa e Whyatt (2013) mostram que a comunicação boca-a-boca influencia a utilização da comunicação eletrônica, e que a atitude em relação à comunicação eWOM modera parcialmente o impacto de adotar essa tecnologia eletrônica pelo viajante. Por fim, Wolny e Mueller (2013) mostraram que o envolvimento e comprometimento com marcas de moda aumentam a motivação das pessoas em se engajar em interações sociais via eWOM.

Varadarajan e Yadav (2002) apontam cinco importantes mudanças no processo de compra decorrentes do surgimento do eWOM: (i) substancial aumento de informações disponíveis sobre preço e atributos de produtos e serviços; (ii) maior facilidade de busca por informações; (iii) melhora da qualidade das informações; (iv) Informações organizadas e estruturadas; e, (v) maior facilidade para comparar e avaliar alternativas consideradas pelo consumidor. 
Outras particularidades do eWOM são a grande variedade de canais para a troca de informações, o anonimato e a confidencialidade do ambiente online permitindo que os consumidores não tenham que se identificar quando procuram ou fornecem informações. Além do mais, desfruta-se da liberdade gerada pela queda das barreiras geográficas e temporais e pela maior disponibilidade deste tipo de mensagem em relação ao tempo que ficam no ar (Gelb \& Sundaram, 2002).

\subsection{Processo de adoção de informações}

Apesar do eWOM constituir em uma forme de troca de informações, o verdadeiro impacto da mensagem recebida pode variar de pessoa pra pessoa (Cheung et al., 2008). Um mesmo conteúdo pode gerar respostas diferentes em diferentes receptores, dependendo da percepção de cada um e de suas experiências (Chaiken \& Eagly, 1976). Esse aspecto elevou o interesse de pesquisadores pela busca de uma maior compreensão de como ocorre o processo de adoção de informações no ambiente virtual, a fim de entenderem como a extensão da influência informativa afeta as pessoas (Cheung et al., 2008).

Na literatura sobre sistemas de informação, vários estudos se baseiam em modelos duplos de processamento para explicar como as pessoas são influenciadas a adotarem ideias e informações. Petty e Cacioppo (1986) afirmam que, apesar de as diferentes teorias de persuasão adotarem diferentes terminologias, postulados, motivos subjacentes e efeitos particulares para explicar as mudanças de atitude, todas acabam enfatizando uma das duas rotas propostas pelo Modelo da Probabilidade de Elaboração (ELM - Elaboration Likelihood Model) para explicar a mudança de atitude.

De acordo com Petty e Cacioppo (1986), o ELM propõe que uma mensagem pode influenciar as atitudes e comportamentos das pessoas de duas maneiras: a primeira, chamada de rota central, postula que a mudança de atitude é resultado de uma avaliação racional e crítica dos argumentos. Mudanças de atitude induzidas pela rota central são relativamente duradouras e previsíveis (Petty, Cacioppo \& Schumann, 1983); a segunda rota, chamada de periférica, argumenta que a mudança de atitude ocorre porque a mensagem foi associada a sinais afetivos positivos ou negativos ou por utilizar-se de uma regra de decisão demasiadamente simples para avaliar a mensagem. Nesse caso, ao invés de refletir sobre o argumento apresentado, a pessoa pode aceitar o mesmo simplesmente porque a fonte da informação é de um especialista. Mudanças de atitude induzidas pelas rotas periféricas são geralmente temporárias e imprevisíveis (Petty et al., 1983).

Em um trabalho seminal, Sussman e Siegel (2003) usaram a base do ELM para propor um modelo teórico de adoção de informação em comunicações por computador com o objetivo de explicar como ocorre o processo de adoção de informações pelas pessoas. O modelo aponta dois fatores-chaves: (1) a qualidade do argumento utilizado; e (2) a credibilidade da fonte. O modelo considera a qualidade dos argumentos como a rota central para a adoção das informações e a credibilidade da fonte como rota periférica. Entretanto, Sussman e Siegal (2003) argumentam que o ELM ignora o papel da utilidade da informação no processo de influência. Sabe-se, porém, que a utilidade é um conceito-chave no processo de adoção de comportamentos. Assim, na elaboração do modelo, os autores acreditam que a utilidade da informação está mais fortemente associada à adoção de informação do que os fatores estudados sob os argumentos do ELM. Dessa forma, defendem que a percepção de utilidade da informação pode ser explicada pelas teorias de influência informacional. Por esses motivos, acreditam que a percepção de utilidade da informação atua como um mediador entre os resultados da influência (por exemplo, a intenção de compra) e as concepções de qualidade do argumento e credibilidade da fonte.

\subsection{Qualidade da informação}

Doll e Torkzadeh (1988) afirmam que a satisfação do usuário de computador está ligada à qualidade da informação, basicamente, por meio do conteúdo, formato, precisão e oportunidade gerada por ela. Particularmente no e-commerce, Cheung et al. (2008) investigaram a influência da qualidade da informação na decisão de usá-lá pelo indivíduo. Uma vez que a publicação de informações esteja aberta a praticamente todos os usuários através da internet, é inevitável o aparecimento de mensagens de baixa qualidade. Dessa forma, segundo esses autores, a força do argumento, a credibilidade da fonte e a confirmação de crenças anteriores influenciam positivamente a qualidade da informação. Já McKinney, Yoon e Zahedi (2002) propõem um modelo de satisfação de consumidores online levando-se em conta três fatores-chave para análise da qualidade da informação: (1) compreensibilidade; (2) confiabilidade; e (3) utilidade.

Por outro lado, usuários da Internet quase nunca leem as páginas da web em detalhes. Ao invés disso, fazem uma busca superficial para encontrar a informação que lhes interessa (Madu \& Madu, 2002). Os usuários querem encontrar as informações que precisam rapidamente e sem grandes esforços (Nah \& Davis, 2002). É importante, portanto, que as comunidades de consumidores online apresentem informações relevantes para seus usuários. Dunk (2004) reforça esse aspecto apontando que a relevância é um importante fator na tomada de decisão, sugerindo que quanto maior o nível de relevância de uma mensagem, maior será a utilidade percebida da informação. Seguindo esse raciocínio, Chung e Koo (2015), ao investigarem a busca por informações turísticas, argumentam que a percepção de valor de uma mensagem quanto vista como relevante pode influenciar positivamente a percepção de utilidade dessa mensagem.

Quando um website não é constantemente atualizado, o conteúdo fornecido não atinge as expectativas dos usuários, deixando de agregar valor a esse conteúdo (Madu \& Madu, 2002). Avaliações antigas podem ser encaradas como desatualizadas pelos usuários, reduzindo sua utilidade. Portanto, quanto maior a atualidade percebida em uma mensagem, maior será a utilidade percebida da informação contida na mesma.

Já a precisão de uma mensagem está ligada a seu nível de confiança. Representa a percepção dos usuários de que a informação apresentada está correta (Wixom \& Todd, 2005). Um dos motivos que leva consumidores a recorrerem ao eWOM é o desejo de reduzir os riscos que envolvem a decisão de compra (Khammash \& Griffiths, 2011). Mensagens imprecisas podem criar 
um efeito contrário ao desejado, causando ainda mais dúvidas em relação ao produto ou serviço pesquisado. Infere-se que quanto maior a precisão de uma mensagem, maior será a utilidade percebida da informação contida na mesma.

Por fim, a abrangência da mensagem está ligada à sua plenitude. Sullivan (1999) sugere que mensagens mais detalhadas atingem uma maior amplitude de usuários, resultando em uma maior probabilidade de conversão e retenção. Logo, quanto mais abrangente for a mensagem, maior será a utilidade percebida da informação contida na mesma. Levando-se em conta as quatro dimensões acima - relevância, atualidade, precisão e abrangência -, associadas à qualidade da informação, enunciam-se as quatro primeiras hipóteses deste estudo:

H1: A percepção de que uma mensagem possui um alto nível de relevância terá um efeito direto e positivo sobre a percepção de utilidade da informação contida na mensagem.

H2: A percepção de que uma mensagem é atual terá um efeito direto e positivo sobre a percepção de utilidade da informação contida na mensagem.

H3: A percepção de que uma mensagem é precisa terá um efeito direto e positivo sobre a percepção de utilidade da informação contida na mensagem.

H4: A percepção de que uma mensagem é abrangente terá um efeito direto e positivo sobre a percepção de utilidade da informação contida na mensagem.

\subsection{Credibilidade da fonte}

Credibilidade da fonte é definida por Chaiken (1980) como sendo a percepção do receptor de uma mensagem acerca da credibilidade do autor da mesma, sem que haja qualquer reflexão sobre a mensagem em si. Também pode ser definida como a medida com que o destinatário de uma mensagem percebe sua fonte como credível, competente e confiável (Petty \& Cacioppo, 1986).

No que diz respeito ao aspecto da influência de informações, mensagens atribuídas a fontes de prestígio geralmente possuem maior grau de aceitação do que àquelas atribuídas a fontes de pouco prestígio (Hovland \& Weiss, 1951). Nesse aspecto, Zhang, Zhang e Yang (2016) mostram que críticas positivas de usuários identificados como fontes de prestígio, ao avaliarem hotéis na internet, influenciam de forma positiva futuras críticas que usuários comuns venham a escrever. Além do mais, a confiabilidade do conteúdo se mostra particularmente relevante, pois a confiança que se forma de uma comunidade online é importante na formação de uma atitude em relação a um produto, como no caso de turistas, que podem ser influenciados por outras mensagens de clientes que eles veem como confiáveis (Sparks, Perkins, \& Buckley, 2013).

Para Cheung et al. (2008), a credibilidade flui pela rota periférica do ELM, em que o receptor pode ser influenciado pela atratividade, simpatia e credibilidade da fonte. Cheung et al. (2008) argumentam que a adoção de informações leva em consideração a credibilidade da fonte por meio de duas dimensões: (1) o nível de especialização da fonte e (2) o nível de confiabilidade da fonte.
Pelo fato de na internet os usuários de comunidades de consumidores online terem liberdade quase ilimitada para publicar e expressar seus sentimentos sem precisar revelar sua identidade, fica a cargo dos leitores das avaliações determinar o grau de especialização e confiabilidade dos colaboradores, a fim de adotar ou rejeitar as informações apresentadas. Se o usuário entender que os comentários postados são provenientes de pessoas com alta credibilidade (altos níveis de especialização e confiabilidade), terá uma maior percepção de utilidade dos comentários (Cheung et al., 2008).

Uma forma de se avaliar a reputação de um usuário se faz por meio da quantidade de avaliações postadas por ele. Outra técnica é perguntar ao leitor se determinada avaliação lhe foi útil. A credibilidade do usuário em questão será então medida pela quantidade de leitores que apontou suas avaliações como úteis (Cheung, Luo, Sia \& Chen 2009).

Assim, em relação à credibilidade da fonte, é possível formular as seguintes hipóteses:

H5: A percepção de que a fonte de uma mensagem é especializada no assunto terá um efeito direto e positivo sobre a percepção de utilidade da informação contida na mensagem.

H6: A percepção de que a fonte de uma mensagem é confiável terá um efeito direto e positivo sobre a percepção de utilidade da informação contida na mensagem.

\subsection{Percepção de utilidade e a Adoção da informação}

A utilidade da informação refere-se à percepção de que o uso dessa informação trará benefícios a quem adotá-la, sendo um importante predecessor da adoção de informações (Davis, 1989). Esse tipo de comportamento é o que mais leva usuários a realizarem buscas em comunidades de consumo (Cheung et al., 2008). Já a adoção da informação é um processo pelo qual as pessoas propositalmente se dispõem a utilizar a informação recebida.

Dentro das comunidades de consumidores online, diversas opiniões e informações relacionadas a produtos, serviços e empresas são apresentadas. Ao analisá-las, os usuários desenvolvem uma percepção individual do quanto essas informações podem ser úteis a eles (Kucukusta, Law, Besbes \& Legohérel, 2015). Logo, quanto maior a percepção de utilidade de uma informação contida em uma comunidade de consumidores online, maior será a intenção de adotá-la (Cheung et al., 2008). Assim:

H7: A percepção de que uma mensagem contém informações de grande utilidade terá um efeito direto e positivo sobre a atitude de um consumidor com relação à adoção das informações.

Com base nas propostas de Cheung et al. (2008) e Sussman e Siegal (2003), a figura 1 apresenta o modelo adotado para este estudo. Assim, no modelo, a qualidade do argumento leva em conta quatro dimensões previstas em Cheung et al. (2008): a relevância, a atualidade, a precisão e a abrangência. $E$ a credibilidade leva em consideração: a especialização da fonte e a sua confiabilidade e como esses dois construtos afetam a utilidade da informação e, consequentemente, a adoção (ou não) da informação. 
Figura 1 - Modelo de adoção de informações e hipóteses da pesquisa

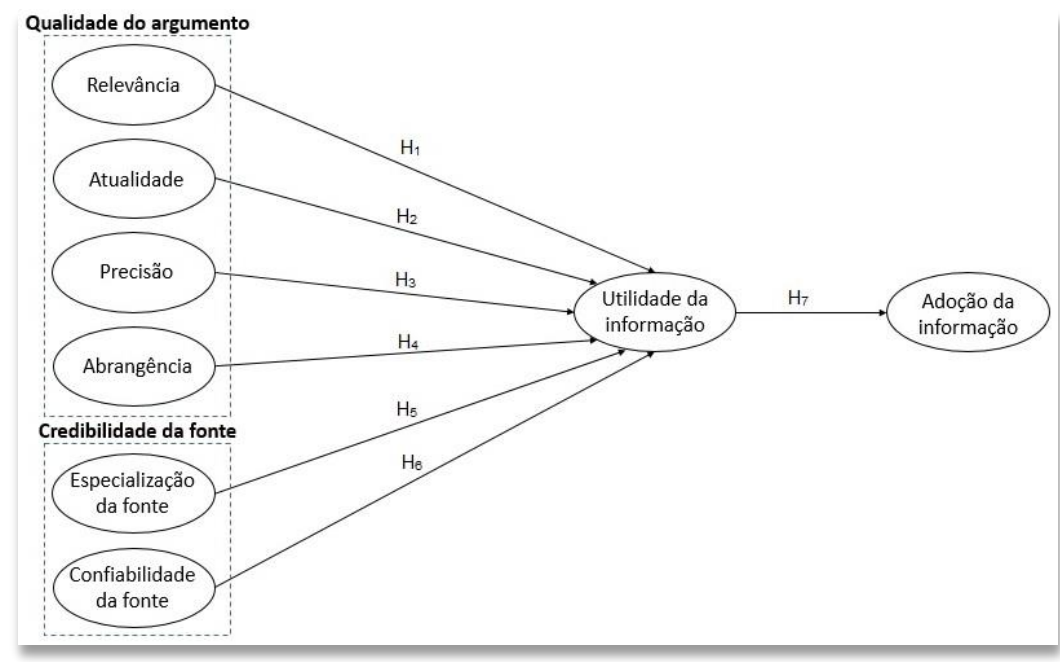

Fonte: Adaptado de Cheung et al. (2008) e Sussman e Siegal (2003).

\section{Metodologia}

Para o teste das hipóteses foi realizada uma cross-sectional survey (Parasuraman, Grewal \& Krishnan, 2006) com a população de interesse, com questionários estruturados, apresentados a consumidores em um único momento no tempo. Os questionários eram auto-administrados (Aaker, Kumar \& Day, 2001) e foram disponíveis em um website de pesquisa (Qualtrics), com os links sendo distribuído por meio de e-mail e redes sociais. Os dados foram analisados por meio de modelagem de equações estruturais, utilizando o software AMOS.

Como o estudo busca investigar os fatores que afetam a adoção de informações em comunidades de consumo online, o modelo adotado foi testado com base em uma comunidade existente, o TripAdvisor, o maior site de viagens do mundo, disponibilizando mais de 150 milhões de avaliações de usuários sobre hotéis, restaurantes e inúmeras atrações turísticas.

\subsection{Operacionalização das Variáveis}

Fez-se uso de escalas (Quadro 1) já elaboradas e testadas na literatura para a medição de todos os construtos envolvidos no modelo proposto. $\mathrm{O}$ instrumento de pesquisa foi composto por 26 itens, sendo medidos via escalas do tipo Likert de 5 pontos, variando de "discordo totalmente" a "concordo totalmente". Os itens incluídos no instrumento de pesquisa foram traduzidos para o português por dois profissionais, com etapas de tradução e retradução para garantir que as escalas em português se aproximassem o máximo possível das originais. Foram realizados dois pré-testes, cada um com 15 pessoas da população de interesse, com o objetivo de refinar os itens e o layout do site utilizado para a pesquisa. No primeiro pré-teste, verificou-se que não havia necessidade de ajustar o layout do questionário. Em relação ao questionário em si e sua compreensão, foram efetuadas algumas sugestões pontuais pelos respondentes. Feitos os ajustes, efetuou-se um segundo pré-teste com outra pequena amostra de 15 pessoas diferente da primeira, em que houve a compreensão correta das questões.

\section{Quadro 1 - Escalas operacionais para cada variável do instrumento de pesquisa}

\begin{tabular}{|c|c|}
\hline Construto & Tipo de escala e medidas operacionais \\
\hline $\begin{array}{l}\text { Relevância } \\
3 \text { itens, adaptada de Citrin } \\
\text { (2001) }\end{array}$ & $\begin{array}{l}\text { REL1: Os comentários postados por usuários no TripAdvisor são relevantes. } \\
\text { REL2: Os comentários postados por usuários no TripAdvisor são adequados. } \\
\text { REL3: Os comentários postados por usuários no TripAdvisor são aplicáveis. }\end{array}$ \\
\hline $\begin{array}{l}\text { Atualidade } \\
3 \text { itens, adaptada de } \\
\text { Wixom e Todd (2005) }\end{array}$ & $\begin{array}{l}\text { UTD1: Os comentários postados por usuários no TripAdvisor são atuais. } \\
\text { UTD2: Os comentários postados por usuários no TripAdvisor são oportunos. } \\
\text { UTD3: Os comentários postados por usuários no TripAdvisor consideram as mais recentes novidades. }\end{array}$ \\
\hline $\begin{array}{l}\text { Precisão } \\
3 \text { itens, adaptada de } \\
\text { Wixom e Todd (2005) }\end{array}$ & $\begin{array}{l}\text { ACC1: Os comentários postados por usuários no TripAdvisor são precisos. } \\
\text { ACC2: Os comentários postados por usuários no TripAdvisor são corretos. } \\
\text { ACC3: Os comentários postados por usuários no TripAdvisor são confiáveis. }\end{array}$ \\
\hline $\begin{array}{l}\text { Abrangência } \\
4 \text { itens, adaptada de } \\
\text { Wixom e Todd (2005) }\end{array}$ & $\begin{array}{l}\text { COMP1: Os comentários postados por usuários no TripAdvisor cobrem suficientemente suas necessidades. } \\
\text { COMP2: Os comentários postados por usuários no TripAdvisor incluem todos os aspectos necessários. } \\
\text { COMP3: Os comentários postados por usuários no TripAdvisor atendem o que você procura. } \\
\text { COMP4: Os comentários postados por usuários no TripAdvisor têm amplitude e profundidade suficientes. }\end{array}$ \\
\hline $\begin{array}{l}\text { Nível de especialização da } \\
\text { fonte } \\
3 \text { itens, adaptada de Wu e } \\
\text { Shaffer (1987) }\end{array}$ & $\begin{array}{l}\text { SEXP1: Os usuários que deixaram comentários no TripAdvisor possuem aptidão para avaliar a qualidade de hotéis, } \\
\text { restaurantes ou atrações turísticas. } \\
\text { SEXP2: Os usuários que deixaram comentários no TripAdvisor possuem bom conhecimento para avaliar a } \\
\text { qualidade de hotéis, restaurantes ou atrações turísticas. } \\
\text { SEXP3: Os usuários que deixaram comentários no TripAdvisor são experts na avaliação de hotéis, restaurantes ou } \\
\text { atrações turísticas. }\end{array}$ \\
\hline
\end{tabular}


Construto

Nível de confiabilidade da

fonte

3 itens, adaptada de Wu e

Shaffer (1987)

Utilidade da informação

3 itens, adaptada de

Bailey e Pearson (1983)

\section{Adoção da informação}

3 itens, adaptada de Wu e

Shaffer (1987)
Tipo de escala e medidas operacionais

SREL1: Os usuários que deixaram comentários no TripAdvisor são fidedignos. SREL2: Os usuários que deixaram comentários no TripAdvisor são confiáveis. SREL3: Os usuários que deixaram comentários no TripAdvisor são sinceros. SREL4: Os usuários que deixaram comentários no TripAdvisor são credíveis.

USE1: Os comentários postados por usuários no TripAdvisor são valiosos.

USE2: Os comentários postados por usuários no TripAdvisor são informativos. USE3: Os comentários postados por usuários no TripAdvisor são úteis.

IA1: Comentários e avaliações positivas postados por usuários no TripAdvisor podem influenciar sua escolha de hotéis, restaurantes ou pontos turísticos a visitar.

IA2: Comentários e avaliações negativas postados por usuários no TripAdvisor podem fazer você desistir de visitar hotéis, restaurantes ou pontos turísticos antes cogitados.

IA3: Sua escolha final de hotéis, restaurantes ou pontos turísticos durante uma viagem leva em consideração os comentários e avaliações postados por usuários no TripAdvisor.

\subsection{Amostra e Procedimentos de Coleta de Dados}

Foi utilizada uma amostragem não probabilística por conveniência (Aaker et al., 2001), obtendo-se 328 respondentes, dos quais 36 foram eliminados por apresentarem dados ausentes ou inconsistentes. Outros 4 foram eliminados por apresentarem idade abaixo de 18 anos, não se encaixando na população de interesse. A coleta de dados ocorreu entre abril e maio de 2014, por meio de questionários auto-administrados respondidos em um site de pesquisas (Qualtrics). Todos os respondentes foram convidados a participar da pesquisa via postagens em redes sociais ou e-mails contendo o link da pesquisa. A amostra final foi composta por 288 questionários válidos, sendo 146 do sexo feminino $(50,7 \%)$ e 142 do sexo masculino (49,3\%). Em relação ao estado civil, 256 (88,9\%) eram formados por solteiros e 32 casados (11,1\%). Já em relação à renda, $62,8 \%$ indicaram renda familiar mensal superior a dez mil reais, enquanto outros $18,1 \%$ afirmaram ter renda familiar entre seis e dez mil reais. A maioria $(77,1 \%)$ possuía entre 18 e 24 anos, enquanto 35 respondentes tinham 30 anos ou mais $(12,2 \%)$. A média de idade observada foi de 23,6 anos. 52,4\% dos respondentes afirmou viajar de duas a três vezes ao ano. $23,3 \%$ uma vez ao ano e $10,1 \%$ mais de 6 vezes ao ano. O principal motivo das viagens é o lazer $(94,1 \%)$, com $3,8 \%$ tendo como principal motivo a viagem a negócios, eventos ou convenções. Em relação ao uso do TripAdvisor, $64,9 \%$ afirmaram já ter usado a plataforma para buscar informações sobre hotéis, restaurantes ou atrações turísticas, enquanto $24,3 \%$ apontaram ter usado a plataforma para compartilhar experiências. O quinto item do questionário pedia que os respondentes apontassem os motivos pelos quais usam o TripAdvisor. O motivo mais apontado foi procurar sugestões de hotéis, restaurantes ou atrações turísticas $(69,8 \%)$.

\section{Resultados}

\subsection{Modelo de Mensuração}

A análise fatorial confirmatória (CFA) foi realizada para testar a validade, unidimensionalidade e confiabilidade das escalas utilizadas no modelo de mensuração. Para avaliar o ajuste do modelo, diversos índices foram utilizados (tanto incrementais quanto absolutos), uma vez que não existe consenso na literatura sobre qual índice (ou conjunto de índices) deve ser utilizado (Hu \& Bentler, 1999). O modelo de mensuração final, com 26 indicadores, apresentou índices de ajuste satisfatórios com um RMSEA de 0,059 (com C.I. de 0,051 até 0,066), CFI de 0,91, IFI de 0,91 , TLI de 0,90 e um valor significativo para índice qui-quadrado $\left(\chi^{2}=538,93\right.$, d.f. $=271, p<0,001, \chi^{2} /$ d.f. $\left.=1,989\right)$.

A validade de face para todas as escalas foi garantida durante o desenvolvimento do instrumento de pesquisa. Para verificar a validade nomológica, foi analisada a matriz de correlação entre os construtos, com todas elas sendo significativas e estando na direção esperada. No que diz respeito à validade convergente, calculou-se a variância extraída média para cada construto (AVE). Todos os valores de AVE estavam entre 0,50 e 0,70, evidenciando a validade convergente das escalas (Tabela 1 ). Com relação à consistência interna e confiabilidade das escalas, todas atenderam aos níveis mínimos de confiabilidade (Fornell \& Larcker, 1981), apresentando valores entre 0,70 e 0,85 tanto para o coeficiente alfa quanto para a confiabilidade composta (Tabela 1). Por fim, todas as variâncias compartilhadas foram inferiores à variância extraída pelos itens que medem os construtos, indicando validade discriminante adequada.

Tabela 1 - Confiabilidade, Confiabilidade Composta e Variância Média Extraída (AVE)

\begin{tabular}{|l|c|c|c|}
\hline \multicolumn{1}{|c|}{ Construct } & Confiabilidade $(\boldsymbol{\alpha})$ & Confiabilidade Composta & AVE \\
\hline Relevância & 0.77 & 0.73 & 0.52 \\
\hline Atualidade & 0.75 & 0.70 & 0.50 \\
\hline Precisão & 0.74 & 0.72 & 0.51 \\
\hline Abrangência & 0.88 & 0.85 & 0.59 \\
\hline Nível de especialização da fonte & 0.76 & 0.73 & 0.50 \\
\hline Nível de confiabilidade da fonte & 0.82 & 0.80 & 0.50 \\
\hline Utilidade da informação & 0.81 & 0.75 & 0.60 \\
\hline Adoção da Informação & 0.74 & 0.71 & 0.53 \\
\hline
\end{tabular}

Fonte: Dados de pesquisa. 


\subsection{Modelo Estrutural}

A modelagem de equações estruturais (SEM) foi utilizada para testar o modelo e as hipóteses. Todos os índices indicaram um bom ajuste do modelo aos dados. $O$ índice qui-quadrado obtido foi estatisticamente significante $\left(\chi^{2}=853,866\right.$, d.f. $\left.=292, p<0,001\right)$. A razão $\chi^{2} /$ d.f. foi de 2,92 , inferior ao valor de 3,0 , como sugerido por Byrne (2010). Os índices de ajuste incrementais ficaram dentro dos padrões da literatura, com CFI de 0,91, IFI de 0,92, TLI de 0,90. O RMSEA de 0,087 (com C.I. de 0,081 até 0,093), também próximo ao que sugere a literatura. Conclui-se que o ajuste do modelo é satisfatório.
Após a verificação do ajuste dos modelos de mensuração e estrutural, foram avaliados os coeficientes estimados para as relações causais entre os construtos (Tabela 2). A verificação de cada uma das hipóteses foi realizada com a análise da magnitude, direção e significância dos coeficientes padronizados estimados por meio do modelo estrutural (Byrne, 2010). O modelo foi capaz de explicar $67 \%$ da variabilidade do construto Utilidade da Informação e $55 \%$ da variância do construto Adoção da Informação, indicando, portanto, que os construtos utilizados como antecedentes explicam um bom nível da variabilidade das variáveis dependentes estudadas.

Tabela 2 - Coeficientes Padronizados Estimados, Hipóteses e Significâncias

\begin{tabular}{|c|c|c|c|}
\hline Relação Proposta & $\begin{array}{l}\text { Coeficiente } \\
\text { Padronizado }\end{array}$ & p-value & $\begin{array}{l}\text { Hipótese } \\
\text { Verificada }\end{array}$ \\
\hline $\mathrm{H}_{1}$ : Relevância $\rightarrow$ Utilidade da Informação & 0,49 & $<0,001$ & Sim \\
\hline $\mathrm{H}_{2}$ : Atualidade $\rightarrow$ Utilidade da Informação & 0,18 & 0,007 & Sim \\
\hline $\mathrm{H}_{3}$ : Precisão $\rightarrow$ Utilidade da Informação & $-0,14$ & 0,035 & Não \\
\hline $\mathrm{H}_{5}$ : Nível de especialização da fonte $\rightarrow$ Utilidade da Informação & $-0,06$ & 0,292 & Não \\
\hline $\mathrm{H}_{6}$ : Nível de confiabilidade da fonte $\rightarrow$ Utilidade da Informação & 0,38 & $<0,001$ & Sim \\
\hline $\mathrm{H}_{7}$ : Utilidade da informação $\rightarrow$ Adoção da Informação & 0,72 & $<0,001$ & Sim \\
\hline
\end{tabular}

Fonte: Dados de pesquisa.

\section{Discussão}

Os resultados apontam que a utilidade da informação exerce grande influência na decisão do consumidor em adotar informações provenientes de comunidades online (H7). Este achado se alinha aos obtidos por Cheung et al. (2008) e indica que, também no contexto brasileiro, os consumidores adotam informações percebidas como úteis por acreditarem que as mesmas trarão benefícios futuros.

Relevância, atualidade, precisão e abrangência foram os construtos utilizados para testar a influência da qualidade da informação, enquanto os níveis de especialização e confiabilidade da fonte foram usados para testar a influência da credibilidade da fonte.

A relevância foi o fator que exerceu maior influência sobre a utilidade percebida da informação $(\mathrm{H} 1)$, reforçando o que Dunk (2004) já havia identificado quando afirma que a relevância é um importante fator na tomada de decisão. Esse resultado sugere que os usuários muitas vezes não possuem paciência ou tempo suficiente para ler todas as avaliações sobre um estabelecimento, comportamento reforçado por Nah e Davis (2002), ao ressaltarem que os usuários querem encontrar as informações que precisam de forma rápida e com o menor esforço possível.

Uma das implicações desse resultado é que uma vez comprovada a influência da relevância sobre a utilidade das informações, as plataformas de eWOM deveriam buscar formas de destacar pontos relevantes das avaliações com o intuito de facilitar o uso e avaliação da informação pelo usuário. É preciso destacar, porém, que os usuários possuem diferentes critérios de avaliação e uma mesma informação pode ser relevante para uns e irrelevante para outros. Incluir um título ou indicar palavras-chave são ferramentas que poderiam facilitar a busca por informações relevantes.

A atualidade também exerceu efeito direto e positivo sobre a utilidade da informação (H2). Entretanto, os resultados aqui encontrados contrariam os resultados de Cheung et al. (2008), que em seu modelo não teve qualquer influência sobre a utilidade da informação. Uma explicação para a confirmação dessa relação no contexto brasileiro pode ser atribuída ao tipo de comunidade investigada. O estudo de Cheung et al. (2008) foi feito em uma comunidade que avalia alimentos enquanto neste estudo a comunidade estaria avaliando experiências relacionadas ao turismo. 0 turismo está altamente ligado à prestação de serviços e sazonalidade, o que exige que as informações relacionadas sejam constantemente atualizadas. Já no que diz respeito a itens de alimentação, a atualidade da informação pode não parecer tão importante para o consumidor, pois dificilmente ao longo do tempo haveria mudança significativas da qualidade dos produtos, ao contrário do que ocorre com serviços, como no caso do turismo. Assim, informações antigas parecem ter efeito na utilidade percebida da informação, o que sugere que o usuário não estaria disposto a adotá-la caso percebesse que a mesma está desatualizada.

Já o efeito da precisão sobre a utilidade da informação (H3) não se confirmou. A possível dificuldade dos respondentes em avaliarem a precisão das mensagens pode ser uma explicação para não se considerar a precisão na avaliação das mensagens. Cheung et al. (2008) afirmam que a percepção de precisão envolve a confirmação de partes da mensagem que o usuário sabe previamente serem verdadeiras ou falsas. Se parte do comentário combinar com o que o usuário já sabe, este estará mais inclinado 
a considerar o resto do comentário como preciso. Os resultados aqui encontrados também se alinham com os obtidos por Cheung et al. (2008).

Já abrangência da mensagem se mostrou um fator de influência positiva sobre a utilidade da informação (H4). Isto é, quanto mais abrangentes e detalhadas forem as informações contidas nas mensagens, maior será a percepção de utilidade da mesma, corroborando resultados anteriores (Cheung et al., 2008).

Dentre os construtos que testavam a credibilidade da fonte, o nível de especialização não apresentou efeito significativo (H5), em linha com Cheung et al. (2008). Assim, também dentro do contexto brasileiro, não foi possível confirmar a influência da especialização sobre a percepção de utilidade da informação. Um dos fatores que torna o eWOM tão influente é exatamente a espontaneidade com que usuários comuns se dispõe a compartilhar as experiências obtidas com produtos ou serviços. Os resultados indicam que os usuários do TripAdvisor não estão em busca da opinião de especialistas, mas sim de opiniões de pessoas comuns como ele. A avaliação de especialistas, que na maioria das vezes possui conotação comercial, pode ser encontrada em outras fontes. Pode-se inferir, portanto, que no eWOM o nível de especialização da fonte não exerce influência positiva sobre a percepção de utilidade, podendo, em contrapartida, exercer influência negativa caso a avaliação seja percebida como comercial.

Já o nível de confiabilidade da fonte foi confirmado como um fator de influência positiva sobre a utilidade da informação (H6), ao contrário do resultado encontrado em Cheung et al. (2008). Os resultados aqui sugerem que os usuários confiam nos julgamentos apresentados em comunidades de consumidores online, apesar de não conhecerem a fonte dos mesmos. A explicação para isso pode estar no fato de os usuários de eWOM perceberem outros usuários como semelhantes. Esse resultado estaria de acordo com a não aceitação de $\mathrm{H} 5$, conforme explicado anteriormente. As avaliações, apesar de muitas vezes pobres do ponto de vista técnico, transmitem com sinceridade a experiência de um consumidor com um determinado produto ou serviço. Pode-se inferir, portanto, que um maior nível de confiabilidade da fonte de uma mensagem aumenta as chances dessa ser percebida como útil pelo receptor.

\subsection{Contribuições teóricas}

Este estudo buscou avaliar a influência da qualidade do argumento da informação e da credibilidade da fonte na utilidade da informação e consequentemente na intenção em adotar a informação. Nesse aspecto, algumas contribuições teóricas podem ser apontadas. Primeiro, uma vez que o estudo dessas relações e sua influencia no eWON ainda são pouco exploradas na literatura de turismo, esta pesquisa amplia o corpo de conhecimento relacionado ao papel do eWON no turismo, mostrando que o argumento da informação e a credibilidade da fonte de fato apresentam influência na utilidade da informação. Segundo, este estudo oferece uma nova luz a como a informação é avaliada, adotada e de seu papel na eWON. Terceiro, os resultados da pesquisa apontaram que os consumidores dão mais importância a opiniões de pessoas comuns do que de experts. Pessoas comuns vivenciam a experiência com o turismo da mesma forma que o leitor comum vivenciaria. Ao descrever sua experiência em uma avaliação online, empresta grande parcela de credibilidade em suas palavras, influenciando de forma positiva a percepção da utilidade da avaliação para o leitor. Por fim, ao investigar o comportamento do consumidor brasileiro, foi possível identificar diferenças de comportamento com usuários em outro contexto, o que de fato ocorreu. Três hipóteses não confirmadas em estudo anterior (Cheung et al., 2008) foram aqui confirmadas: $\mathrm{H} 2$ (Atualidade $\rightarrow$ Utilidade da Informação), H4 (Abrangência $\rightarrow$ Utilidade da Informação) e H6 (Nível de confiabilidade da fonte $\rightarrow$ Utilidade da Informação). Essas diferenças sugerem que o consumidor brasileiro avalia as mensagens de forma diferente do consumidor chinês (contexto de aplicação do estudo de Cheung et al., 2008). Isso abre uma janela de discussão sobre a diferença de percepção do que é efetivamente importante no processo de adoção da informação por consumidores em comunidades online e nas diferenças de comportamento em diferentes países.

\section{Conclusões}

Os resultados aplicados na prática podem ajudar os gestores de plataformas de eWOM a aumentar a qualidade da informação e o fluxo de usuários de suas páginas. De forma semelhante, empresas interessadas em complementar sua promoção através deste tipo de comunicação também podem se beneficiar dos resultados, analisando mais claramente o tipo de informação que seu consumidor busca.

Nesse sentido, foi verificado o grande efeito da relevância sobre a utilidade da informação. Com isso, as plataformas devem se esforçar para destacar informações relevantes das avaliações com o objetivo de reter a atenção dos usuários, como por exemplo com a inclusão de um campo com palavras-chave contidas em cada avaliação. Dessa forma, o usuário que busca comentários relacionados ao atendimento de um hotel ou restaurante, por exemplo, poderia filtrar os comentários, a fim de ler apenas os que Ihe interessam ou são relevantes.

A abrangência também provou exercer influência sobre a utilidade da informação. Os usuários desejam obter o maior número possível de informações disponíveis sobre o produto ou serviço buscado. Apresentar um resumo intuitivo dos principais critérios avaliados em um estabelecimento (uma classificação de 1 a 5 estrelas, por exemplo) pode ser uma forma de destacar a abrangência das avaliações.

Os estabelecimentos avaliados nas plataformas de eWOM podem (e devem) acompanhar constantemente suas páginas nessas plataformas para garantir que as informações básicas como endereço, telefone, menu e faixa de preço estejam sempre atualizadas. Fora isso, o eWOM é uma excelente forma de se obter o feedback de seus clientes a respeito dos produtos e serviços oferecidos, podendo apresentar resultados melhores do que pesquisas de satisfação realizadas diretamente com os consumidores.

Por fim, os resultados indicam que os usuários do TripAdvisor não estão em busca da opinião de especialistas, mas sim de opiniões de pessoas comuns como ele. Conclui-se que, no meio da eWOM, o nível de especialização da fonte não exerce necessariamente uma influência positiva sobre a percepção de utilidade das 
mensagens, podendo exercer influência negativa caso a avaliação seja percebida como comercial. As avaliações, apesar de muitas vezes pobres do ponto de vista técnico, transmitem com sinceridade a experiência de um consumidor com um determinado produto ou serviço. Assim, os leitores dessas avaliações acreditam que poderiam vivenciar experiências semelhantes, sejam elas positivas ou negativas, caso venham a adquirir o produto ou serviço em questão.

O presente estudo limitou-se a investigar apenas uma comunidade de consumidores online no setor de turismo, o TripAdvisor. Não se pode afirmar que usuários de outras comunidades possuam os mesmos hábitos. Como sugestão de futuros estudos, o modelo poderia ser testado em plataformas de eWOM focadas em outras áreas de consumo. Seria interessante verificar se as relações entre os construtos aqui utilizados são específicas ou não da área do turismo. Além disso, seria interessante verificar as diferenças dos resultados entre aqueles que viajam a negócios e a lazer.

Por fim, sugere-se também que o modelo possa ser testado usando novas dimensões da qualidade dos argumentos, tais como o formato (Wixom \& Todd, 2005) e a compreensibilidade (Srinivasan, 1985), para entender melhor outros fatores que possam influenciar a percepção de utilidade da informação e sua adoção (ou não).

Em relação às limitações do estudo, a amostragem não probabilística pode levar a resultados que podem não ser generalizáveis. A amostra por conveniência foi realizada em grande parte com alunos de uma universidade particular resultou em um percentual elevado de respondentes jovens e com renda familiar acima de dez mil reais mensais. Uma amostra mais diversificada em relação à idade, renda e ocupação dos respondentes poderia esclarecer a influência da informação no comportamento do consumidor.

\section{Referências}

Aaker, D., Kumar, V., \& Day, G. (2001). Pesquisa de Marketing. São Paulo: Atlas.

Bailey, J. E., \& Pearson, S. W. (1983). Development of a tool for measuring and analyzing computer user satisfaction. Management Science, 29(5), 530-545.

Byrne, B. M. (2010). Structural Equation Modeling with AMOS: Basic Concepts, Applications and Programming. (2a ed.). New York: Routledge.

Chaiken, S. (1980). Heuristic versus systematic information processing and the use of source versus message cues in persuasion. Journal of Personality and Social Psychology, 39(5), 752-766.

Chaiken, S. \& Eagly, A.H. (1976). Communication modality as a determinant of message persuasiveness and message comprehensibility. Journal of Personality and Social Psychology, 34(4), 605-614.

Chan, Y., \& Ngai, E. (2011). Conceptualising electronic word of mouth activity: An input-process-output perspective. Marketing intelligence \& Planning, 9(5), 488-516.

Chen, Z., \& Lurie, N.H. (2013). Temporal Contiguity and Negativity Bias in the Impact of Online Word of Mouth. Journal of Marketing Research. 50(4), 463-476.

Cheung, C. M., Lee, M. K. Rabjohn, N. (2008). The Impact of electronic word-of-mouth: The adoption of online opinions in online customer communities. Internet Research, 18(3), 229-247.

Cheung, M., Luo, C., Sia, C., \& Chen, H. (2009). Credibility of Electronic Word-of-Mouth: Informational and Normative Determinants of On-line Consumer Recommendations. International Journal of Electronic Commerce. 13(4), 9-38.
Chung, N., \& Koo, C. (2015). The use of social media in travel information search. Telematics and Informatics, 32(2), 215-229.

Citrin, A. V. (2001). Information quality perceptions: the role of communication media characteristics. Information and Learning, 17, 1-143 Doll, W.J., \& Torkzadeh, G. (1988). The measurement of end-user computing satisfaction. MIS Quarterly, 12(2), 259-74.

Davis, F. D. (1989). Perceived usefulness, perceived ease of use, and user acceptance of information technology. MIS Quarterly, 13(3), 319-340.

Dunk, A. S. (2004). Product life cycle cost analysis: the impact of customer profiling, competitive advantage, and quality of IS information. Management Accounting Research. 15(4), 401-414.

Fornell, C. \& Larcker, D. F. (1981). Evaluating Structural Equation Models with Unobservable Variables and Measurement Error. Journal of Marketing Research, 18, 39-50.

Gelb, B. D., \& Sundaram, S. (2002). Adapting to "word of mouse". Business Horizons Greenwich, 45(4), 21-5,

Goldsmith, R. E. (2006). Electronic Word-of-Mouth. In: Khosrow-Pour, M. Encyclopedia of E-Commerce, E-Government, and Mobile Commerce. Pennsylvania: IGI Global.

Goldsmith, R. E. \& Horowitz, D. (2006). Measuring motivations for online opinion seeking. Journal of Interactive Advertising, 6(2), 1-16.

Hennig-Thurau, T., Gwinner, K., Walsh, G., \& Gremler, D. (2004). Electronic word-of-mouth via consumer-opinion platforms: what motivates consumers to articulate themselves on the Internet? Journal of Interactive Marketing, 18(1), 38-52.

Hovland, C. I. \& Weiss, W. (1951). The Influence of Source Credibility on Communication Effectiveness. The Public Opinion Quarterly, 15(4), 635650.

Hu, L. \& Bentler, P. M. (1999). Cutoff criteria for fit indexes in covariance structure analysis: conventional criteria versus new alternatives. Structural Equation Modeling, 6(1), 1-55.

Hudson, S., Roth, M.S., Madden, T.J., \& Hudson, R. (2015). The effects of social media on emotions, brand relationship quality, and word of mouth: An empirical study of music festival attendees. Tourism Management, 47 (April) 68-76.

Katz, E., \& Lazarsfeld, P. F. (1955). Personal influence: the part played by people in the flow of mass communications. Illinois: The Free Press.

Khammash, M., \& Griffiths, G. H. (2011). 'Arrivederci CIAO.com, Buongiorno Bing.com' - Electronic word-of-mouth (eWOM), antecedences and consequences. International Journal of Information Management, 31(1), 82-87.

Kozinets, R. V., De Valck, K., Wojnicki, A.C., \& Wilner, S.J. (2010). Networked Narratives: Understanding Word-of-Mouth Marketing in Online Communities. Journal of Marketing. 74(2), 71-89.

Kucukusta, D., Law, R., Besbes, A., \& Legohérel, P. (2015). Re-examining perceived usefulness and ease of use in online booking: the case of Hong Kong online users. International Journal of Contemporary Hospitality Management, 27(2), 185-198.

Lee, M.K., Cheung, C.M., Lim, K.H., \& Sia, C.L. (2006). Understanding customer knowledge sharing in web-based discussion boards: an exploratory study. Internet Research, 16(3), 289-303.

Liang, S.W., Ekincia, Y., Occhiocupoa, N., \& Whyatt, G. (2013). Antecedents of travellers" electronic word-of-mouth communication. Journal of Marketing Management. 29(5/6), 584-606.

Luo, Q., \& Zhong, D. (2015). Using social network analysis to explain communication. Tourism Management, 46 (February), 274-282.

Madu, C. N., \& Madu, A. A. (2002). Dimensions of e-quality. International Journal of Quality and Reliability Management. 19(3), 246-258.

Mcdonough, M. (1997). Frequently asked questions: virtual communities. Artigo preparado para o Thomson Virtual Communities Laboratory.

Mckinney, V., Yoon, K., \& Zahedi, F. M. (2002). The measurement of webcustomer satisfaction: an expectation and disconfirmation approach. Information Systems Research, 13(3), 296-315.

Michaelidou, N., Siamagka, N.T. \& Christodoulides, G. (2011). Usage, barriers and measurement of social media marketing: An exploratory investigation of small and medium B2B brand. Industrial Marketing Management, 40(7), 1153-1159. 
Munar A. M., \& Jacobsen, J.K.S. (2014). Motivations for sharing tourism experiences through social media Tourism Management, 43 (August), 46-54.

Nah, F., \& S. Davis (2002). HCl Research Issues in Electronic Commerce. Journal of Electronic Commerce Research, 3(3), 98-113.

Parasuraman, A., Grewal, D., \& Krishnan, R. (2006). Exploring marketing research. (2a ed.) South Western College Pub.

Park, C., \& Lee, T. (2009). Information direction, website reputation and eWOM effect: A moderating role of product type. Journal of Business Research, 62(1), 61-67.

Petty, R. E. \& Cacioppo, J. T. (1986). Communication and Persuasion: Central and Peripheral Routes to Attitude Change. New York: SpringerVerlag.

Petty, R. E., Cacioppo, J. T. \& Schumann D. (1983). Central and peripheral routes to advertising effectiveness: the moderating role of involvement. Journal of Consumer Research, 10(2), 135-146.

Srinivasan, A. (1985). Alternative measures of systems effectiveness: associations on consumers' online choices. Journal of Retailing, 80(2), 159169.

Sparks, B.A., Perkins, H.E., \& Buckley, R. (2013). Online travel reviews as persuasive communication: The effects of content type, source, and certification logos on consumer behavior. Tourism Management, 39 (December), 1-9.

Sullivan, C. (1999). Marketing the web in other media. Editor \& Publisher, 132(9), 30.

Sussman, S. W., \& Siegel, W. S. (2003). Informational influence in organizations: an integrated approach to knowledge adoption. Information Systems Research, 14(1), 47-65.

Varadarajan, P. \& Yadav, M. (2002). Marketing strategy and the internet: an organizing framework. Journal of Academy of Marketing Science, 30(4), 296-312.

Vieira, V. A., Matos, C. A., \& Slongo, L. A. (2009). Avaliação das relações entre qualidade de serviço do site, satisfação, valor percebido, lealdade e boca a boca por meio de um modelo teórico. Revista de Administração, 44(2), 131-146.

Wixom, B. H., \& Todd, P. A. (2005). A theoretical integration of user satisfaction and technology acceptance. Information Systems Research, 16(1), 85-102.

Wolny, J., \& Mueller, C. (2013). Analysis of fashion consumers' motives to engage in electronic word-of-mouth communication through social media platforms. Journal of Marketing Management, 29(5/6), 562-583.

$\mathrm{Wu}$, C. \& Shaffer, D. (1987). Susceptibility to persuasive appeals as a function of source credibility and prior experience with the attitude object. Journal of Applied Psychology, 52(4), 677-688.

Ya, Y., Vadakkepatt, G., \& Joshi, A. (2015). A meta-analysis of electronic word-of-mouth elasticity. Journal of Marketing. 79(2), 19-39.

Ye, Q., Law, R., Gu, B., Chen, W. (2011). The influence of user-generated content on traveler behavior: An empirical investigation on the effects of e-word-of-mouth to hotel online bookings. Computers in Human Behavior, 27(2), 634-639.

Zhang, Z., Zhang, Z., \& Yang, Y. (2016). The power of expert identity: How website-recognized expert reviews influence travelers' online rating behavior. Tourism Management, 55 (August), 15-24.

Recebido: 14.03.2016

Reenviado: 15.07.2016

Aceite: 25.07.2016 